\title{
Efficient Implementation of a Dimensionality Reduction Method Using a Complex Moment-Based Subspace
}

\author{
Takahiro Yano \\ University of Tsukuba \\ Tsukuba, Japan \\ yano.takahiro.fp@u.tsukuba.ac.jp \\ Akira Imakura \\ University of Tsukuba \\ Tsukuba, Japan \\ imakura@cs.tsukuba.ac.jp
}

\author{
Yasunori Futamura \\ University of Tsukuba \\ Tsukuba, Japan \\ futamura@cs.tsukuba.ac.jp \\ Tetsuya Sakurai \\ University of Tsukuba \\ Tsukuba, Japan \\ sakurai@cs.tsukuba.ac.jp
}

\begin{abstract}
Dimensionality reduction methods are widely used for processing data efficiently. Recently Imakura et al. proposed a novel dimensionality reduction method using a complex moment-based subspace. Their method can use more eigenvectors than the existing matrix trace optimization-based methods which explains its reported higher precision. However, the computational complexity is also higher than that of the existing methods, in particular for the nonlinear kernel version. To reduce the computational complexity, we propose a practical parallel implementation of the method by introducing the Nyström approximation. We evaluate the parallel performance of our implementation using the Oakforest-PACS supercomputer.
\end{abstract}

\section{CCS CONCEPTS}

- Computing methodologies $\rightarrow$ Massively parallel algorithms; Spectral methods.

\section{KEYWORDS}

dimensionality reduction, complex moment-based method, parallel computing

\section{ACM Reference Format:}

Takahiro Yano, Yasunori Futamura, Akira Imakura, and Tetsuya Sakurai. 2021. Efficient Implementation of a Dimensionality Reduction Method Using a Complex Moment-Based Subspace. In The International Conference on High Performance Computing in Asia-Pacific Region (HPCAsia 2021), January 20 22, 2021, Virtual Event, Republic of Korea. ACM, New York, NY, USA, 7 pages. https://doi.org/10.1145/3432261.3432267

\section{INTRODUCTION}

Currently, big data analysis is an important technology for mining data and knowledge discovery. To process enormous amounts of

Permission to make digital or hard copies of all or part of this work for personal or classroom use is granted without fee provided that copies are not made or distributed for profit or commercial advantage and that copies bear this notice and the full citation on the first page. Copyrights for components of this work owned by others than ACM must be honored. Abstracting with credit is permitted. To copy otherwise, or republish, to post on servers or to redistribute to lists, requires prior specific permission and/or a fee. Request permissions from permissions@acm.org.

HPCAsia 2021, January 20-22, 2021, Virtual Event, Republic of Korea

(c) 2021 Association for Computing Machinery.

ACM ISBN 978-1-4503-8842-9/21/01 . .\$15.00

https://doi.org/10.1145/3432261.3432267 data, parallel computing is necessary. Therefore, the importance of parallel data processing methods has been increasing.

Most of the meaningful information of a dataset is usually embedded in a lower-dimensional space. To process data efficiently, dimensionality reduction methods are widely used for data analysis.

Dimensionality reduction methods based on matrix trace optimization, such as unsupervised methods like principal component analysis (PCA) [11] and locality preserving projections (LPP) [5] and supervised methods like discriminant analysis and its variants [2, 3, 9, 14], are widely used. In these methods, a linear map is obtained by computing a small number of eigenvectors of an eigenvalue problem. However, valuable information may be dropped because a small number of eigenvectors is used.

Nonlinear dimensionality reduction improves on linear dimensionality reduction methods by using a nonlinear map and kernel trick [13]. A Gram matrix is introduced to reduce the computational complexity by avoiding direct computation of mapped data. However, the size of the Gram matrix equal to the number of samples, which becomes problematic when this number is very large.

Recently, Imakura et al. [6] proposed a dimensionality reduction method called Complex Moment-based Supervised Eigenmap, abbreviated as CMSE, which combines matrix trace optimization and a squared error term. CMSE can use more eigenvectors than conventional matrix trace optimization-based dimensionality reduction methods. As a result, it is reported that CMSE achieves higher accuracy than the existing methods. CMSE constructs a complex moment-based subspace and then generates a linear map from the subspace. In this method, a very large computational complexity is required for constructing the subspace. Thus, this large computation time is required in practice.

Complex moment-based subspace was originally proposed for an eigensolver by Sakurai and Sugiura [12]. These types of eigensolvers have been attracting much attention because of their high scalability. Parallel performance of improved methods on large computational systems has been reported, e.g., [7].

In this paper, we propose a practical parallel implementation of the kernel version of CMSE (K-CMSE). The most time-consuming parts of K-CMSE are handling the Gram matrix and constructing the complex moment-based subspace. The proposed implementation uses the Nyström approximation to reduce the computational and space complexity for handling the Gram matrix. The proposed implementation is also based on the hierarchical parallelism of 
constructing the complex moment-based subspace to achieve high parallel scalability.

Instead of handling the Gram matrix directly, we introduce the Nyström approximation [15] to reduce computational and space complexity. Appropriate assignment of Message Passing Interface (MPI) ranks and communicators is expected to allow parallel implementation to scale well because of the hierarchical parallelism of CMSE.

The remainder of this manuscript is organized as follows. In Section 2, we briefly describe the existing dimensionality reduction methods based on matrix trace optimization, CMSE and K-CMSE. In Section 3, we propose an efficient parallel implementation of K-CMSE. Parallel performance of the proposed implementation is evaluated by numerical experiments in Section 4. Finally, we conclude the paper in Section 5.

\section{A COMPLEX MOMENT-BASED SUPERVISED EIGENMAP FOR DIMENSIONALITY REDUCTION}

In this section, we briefly introduce a complex moment-based supervised eigenmap (CMSE) for dimensionality reduction.

\subsection{Dimensionality Reduction based on Matrix Trace Optimization}

Let $m$ and $n$ be the number of features and samples, respectively. Consider transforming dataset $X=\left\{\boldsymbol{x}_{1}, \ldots, \boldsymbol{x}_{n}\right\} \in \mathbb{R}^{m \times n}$ into a lower-dimension representation $Y=\left\{\boldsymbol{y}_{1}, \ldots, \boldsymbol{y}_{n}\right\} \in \mathbb{R}^{\ell \times n}$ while preserving the properties of the original dataset, where $\ell<m$.

Linear dimensionality reduction methods construct a linear map function $B \in \mathbb{R}^{m \times \ell}$ and map the original data as

$$
Y=B^{\mathrm{T}} X
$$

Let $\left(A_{1}, A_{2}\right)$ be a matrix pencil given in dimensionality reduction methods based on matrix trace optimization. Here, $A_{1} \in \mathbb{R}^{m \times m}$ is real symmetric, and $A_{2} \in \mathbb{R}^{m \times m}$ is real symmetric and positive definite. The linear map $B$ is obtained by solving the following minimization or maximization problem:

$$
\begin{gathered}
\min _{B \in \mathbb{R}^{m \times \ell}} \operatorname{Tr}\left(B^{\mathrm{T}} A_{1} B\right) \text { or } \max _{B \in \mathbb{R}^{m \times \ell}} \operatorname{Tr}\left(B^{\mathrm{T}} A_{1} B\right) \\
\text { s.t. } B^{\mathrm{T}} A_{2} B=I,
\end{gathered}
$$

and can be computed by solving the following generalized eigenvalue problem:

$$
A_{1} \boldsymbol{r}_{i}=\lambda_{i} A_{2} \boldsymbol{r}_{i}
$$

$B=\left[\boldsymbol{r}_{1}, \ldots, \boldsymbol{r}_{\ell}\right]$

\subsection{Basic Concepts}

As shown in Section 2.1, the existing dimensionality reduction methods use $\ell$ eigenvectors for the map function $B$. Instead, to achieve high recognition performance, the basic concepts of CMSE as follows:

- CMSE uses the same matrices $A_{1}$ and $A_{2}$ as used in some existing dimensionality reduction methods, e.g., LPP and LFDA.
- CMSE uses a complex moment-based subspace

$$
\begin{aligned}
& \mathcal{S}_{\Omega}=\mathcal{R}(S), \quad S=\left[S_{0}, S_{1}, \ldots, S_{M-1}\right], \\
& S_{k}:=\frac{1}{2 \pi \mathrm{i}} \oint_{\Gamma} z^{k}\left(z A_{2}-A_{1}\right)^{-1} A_{2} V \mathrm{~d} z,
\end{aligned}
$$

including multiple eigenvectors to preserve more data properties than the existing methods. Here, $L, M \in \mathbb{N}_{+}, V \in$ $\mathbb{R}^{m \times L}$, and $\Gamma$ is a positively oriented Jordan curve around a given interval $\Omega=[a, b]$. Here, we assume that only the target eigenvalues are present inside $\Gamma$.

- CMSE provides a minimization problem

$$
\begin{aligned}
& \min _{B=\left[\boldsymbol{b}_{1}, \boldsymbol{b}_{2}, \ldots, \boldsymbol{b}_{\ell}\right], \boldsymbol{b}_{i} \in \mathcal{S}_{\Omega}} E(B) \quad \text { s.t. } \quad B^{\mathrm{T}} A_{2} B=I, \\
& E(B)=(1-\mu) \operatorname{Tr}\left(B^{\mathrm{T}} f\left(A_{1}\right) B\right)+\mu\left\|Z-B^{\mathrm{T}} X\right\|_{\mathrm{F}}^{2},
\end{aligned}
$$

whose objective function $E(B)$ combines a matrix trace derived from dimensionality reduction methods and a squared error in a straightforward manner using the ground truth data $Z$ such as the ridge regression. Here, $\mu \in[0,1]$ is a weight parameter for both terms, and $f(\cdot)$ is a (meromorphic) weight function of each eigenvector for minimization.

\subsection{A Practical Algorithm}

To solve the minimization problem (3), we first construct an $A_{2}$ orthonormal basis of the complex moment-based subspace (2). In practice, the contour integral in (2) is approximated by a numerical integration rule such as the $N$-point trapezoidal rule, as follows:

$$
\begin{aligned}
\widehat{S} & =\left[\widehat{S}_{0}, \ldots, \widehat{S}_{M-1}\right], \\
S_{k} & \approx \widehat{S}_{k}=2 \sum_{j=1}^{N / 2} \operatorname{Re}\left(\omega_{j} z_{j}^{k}\left(z_{j} A_{2}-A_{1}\right)^{-1} A_{2} V\right),
\end{aligned}
$$

where $\left(z_{j}, \omega_{j}\right)=\left(\bar{z}_{j+N / 2}, \bar{\omega}_{j+N / 2}\right), j=1,2, \ldots, N / 2$ are the integral points and the corresponding weights, respectively.

An $A_{2}$-orthonormal basis of $\mathcal{R}(\widehat{S})$ is constructed by a low-rank approximation of $\widehat{S}$ with a singular value decomposition on an $A_{2}$-inner product:

$$
\begin{aligned}
& \widehat{S}=\left[\widehat{U}, \widehat{U}^{\prime}\right]\left[\begin{array}{ll}
\widehat{\Sigma} & \\
& \widehat{\Sigma}^{\prime}
\end{array}\right]\left[\begin{array}{c}
\widehat{W}^{\mathrm{T}} \\
\widehat{W}^{\prime \mathrm{T}}
\end{array}\right] \approx \widehat{U} \widehat{\Sigma} \widehat{W}^{\mathrm{T}}, \\
& \widehat{U}^{\mathrm{T}} A_{2} \widehat{U}=I, \quad \widehat{W}^{\mathrm{T}} \widehat{W}=I,
\end{aligned}
$$

to improve the numerical stability. Here, $\widehat{\Sigma}$ is a diagonal matrix whose diagonal entries are the $d$ largest singular values where $d$ is determined by a threshold $\delta>0$ as $\sigma_{\widehat{d}} / \sigma_{1} \geq \delta>\sigma_{\widehat{d}+1} / \sigma_{1}$ ( $\sigma_{1} \geq \sigma_{2} \geq \cdots \geq \sigma_{L M}$ are the singular values). The columns of $\widehat{U}, \widehat{W}$ are the corresponding left/right singular vectors. Then, we set $B=\widehat{U} \widehat{C}$ with $\widehat{C} \in \mathbb{R}^{d \times \ell}$.

Using the relationships of a matrix trace and the Frobenius norm, the minimization problem (3) is written as

$$
\begin{aligned}
& \min _{\widehat{C} \in \mathbb{R}^{\widehat{d} \times \ell} \|}\left\|\left[\begin{array}{c}
\mu^{1 / 2} Z^{\mathrm{T}} \\
O
\end{array}\right]-\left[\begin{array}{c}
\mu^{1 / 2} X^{\mathrm{T}} \widehat{U} \\
(1-\mu)^{1 / 2} f(\widehat{T})^{1 / 2}
\end{array}\right] \widehat{C}\right\|_{\mathrm{F}}^{2} \\
& \text { s.t. } \quad \widehat{C}^{\mathrm{T}} \widehat{C}=I,
\end{aligned}
$$


Algorithm 1 Complex moment-based supervised eigenmap for dimensionality reduction

Input: Training Dataset: $X \in \mathbb{R}^{m \times n}, Z \in \mathbb{R}^{\ell \times n}$ and parameters $L, M, N \in \mathbb{N}_{+}, \delta \in \mathbb{R}, V \in \mathbb{R}^{m \times L},\left(z_{j}, \omega_{j}\right)$ for $j=$ $1, \ldots, N / 2, \Omega=[a, b], \mu, f(\cdot)$.

Output: Linear eigenmap $B \in \mathbb{R}^{m \times \ell}$

1: Construct matrix pencil $\left(A_{1}, A_{2}\right)$ from training dataset $X$ and $Z$ if required.

2: Compute a low rank approximation of $\widehat{S}$ by using a threshold $\delta$.

3: Compute $\widehat{S}_{k}$ and set $\widehat{S}=\left[\widehat{S}_{0}, \ldots, \widehat{S}_{M-1}\right] \widehat{S}=$ $\left[\widehat{U}, \widehat{U}^{\prime}\right]\left[\widehat{\Sigma}, O ; O, \widehat{\Sigma}^{\prime}\right]\left[\widehat{W}^{\mathrm{T}} ; \widehat{W}^{\prime \mathrm{T}}\right] \approx \widehat{U} \widehat{\Sigma} \widehat{W}^{\mathrm{T}}$

s.t. $\widehat{U}^{\mathrm{T}} A_{2} \widehat{U}=I$

4: Solve UOP problem (4) and set $B=\widehat{U} \widehat{C}$.

where $\widehat{T}=\widehat{U}^{\mathrm{T}} A_{1} \widehat{U}$. A minimization problem with an orthogonal constraint (4) is called the unbalanced orthogonal Procrustes (UOP) problem, which is solved using an iterative method $[1,10]$.

The algorithm of CMSE is summarized in Algorithm 1. For details, refer to [6].

\subsection{Kernel Version of CMSE}

Consider a nonlinear dimensionality reduction using a nonlinear function $\phi(\cdot)$ and kernel trick.

Nonlinear dimensionality reduction methods transform the original dataset $X$ into $\phi(X):=\left\{\phi\left(\boldsymbol{x}_{1}\right), \ldots, \phi\left(\boldsymbol{x}_{n}\right)\right\}$. The dimensionality of $\phi(X)$ is then reduced using a nonlinear map $\widetilde{B}$ as

$$
Y=\widetilde{B}^{\mathrm{T}} \phi(X) .
$$

By using a linear map $\widehat{B} \in \mathbb{R}^{n \times \ell}, \widetilde{B}$ is represented as $\widetilde{B}=\phi(X) \widehat{B}$.

The generalized eigenvalue problem (1) is transformed by using the Gram matrix of $\phi(X), K:=\phi(X)^{\mathrm{T}} \phi(X)$, as

$$
K A_{1}^{\prime} K \boldsymbol{u}=\lambda K A_{2}^{\prime} K \boldsymbol{u}, \quad \boldsymbol{u} \in \mathbb{R}^{n} \backslash\{\mathbf{0}\},
$$

where the $n \times n$ matrices $A_{1}^{\prime}$ and $A_{2}^{\prime}$ satisfy

$$
A_{1}=X A_{1}^{\prime} X^{\mathrm{T}}, \quad A_{2}=X A_{2}^{\prime} X^{\mathrm{T}} .
$$

The matrices $A_{1}^{\prime}$ and $A_{2}^{\prime}$ are usually sparse matrices; however, $K A_{1}^{\prime} K$ and $K A_{2}^{\prime} K$ become dense matrices. Therefore, we should avoid solving the generalized eigenvalue problem (5) directly when $n$ is very large. Define $\boldsymbol{u}^{\prime}:=K \boldsymbol{u}$, and suppose $K$ is nonsingular. The generalized eigenvalue problem (5) can then be transformed as

$$
A_{1}^{\prime} \boldsymbol{u}^{\prime}=\lambda A_{2}^{\prime} \boldsymbol{u}^{\prime}
$$

Let $B^{\prime} \in \mathbb{R}^{n \times \ell}$ be a linear map obtained by CMSE given with the matrices $A_{1}^{\prime}, A_{2}^{\prime} . \widehat{B}$ is then represented by

$$
\widehat{B}=K^{-1} B^{\prime} \text {. }
$$

In general, $K$ is not necessarily nonsingular. Let

$$
K=\left[\begin{array}{ll}
U_{K} & U_{K}^{\prime}
\end{array}\right]\left[\begin{array}{cc}
\Sigma_{K} & \\
& \Sigma_{K}^{\prime}
\end{array}\right]\left[\begin{array}{c}
U_{K}^{\mathrm{T}} \\
U_{K}^{\prime \mathrm{T}}
\end{array}\right] \approx U_{K} \Sigma_{K} U_{K}^{\mathrm{T}}
$$

be a low rank approximation $K$ with a threshold; then the (pseudo)inverse of $K$ is approximated as $U_{K} \Sigma_{K}^{-1} U_{K}^{\mathrm{T}}$.

The algorithm of the kernel version of CMSE (K-CMSE) is shown in Algorithm 2.

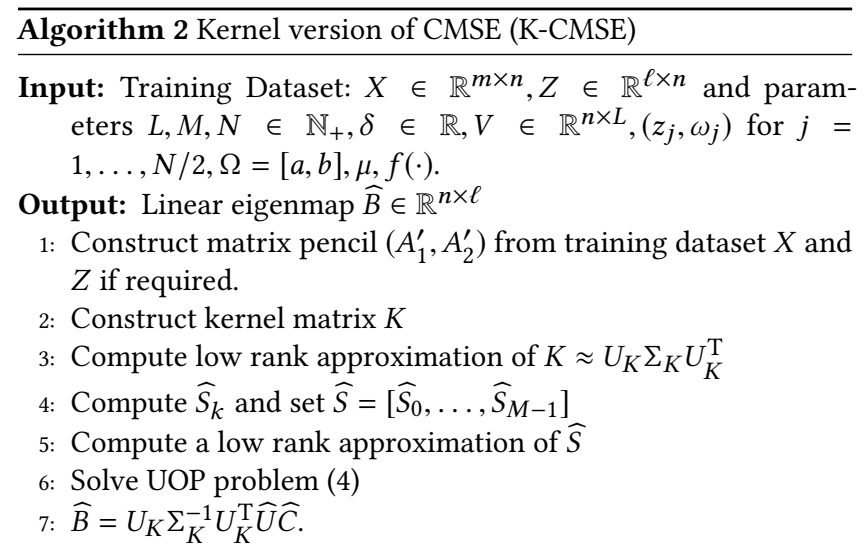

\section{EFFICIENT IMPLEMENTATION}

In this section, we propose a practical parallel implementation of K-CMSE.

\subsection{Handling Large Kernel Matrix with Nyström Approximation}

In K-CMSE, handling $K$ directly is problematic in terms of both computational complexity and memory consumption. To avoid this, we use the Nyström approximation [15] of $K$.

Define a subset of dataset $\widetilde{X}:=\left\{\boldsymbol{x}_{i} \mid i \in \mathcal{I}\right\}, \mathcal{I} \subset\{1, \ldots, n\},|\mathcal{I}|=$ $s<n$, sometimes referred as a landmark. We partition $K$ as

$$
K=\left[\begin{array}{cc}
\tilde{K} & L^{\mathrm{T}} \\
L & *
\end{array}\right]
$$

where $\tilde{K}$ is a kernel matrix of $\tilde{X}$. We also define $C \in \mathbb{R}^{n \times s}$ as

$$
C \equiv\left[\begin{array}{c}
\tilde{K} \\
L
\end{array}\right]
$$

The Nyström approximation approximates the eigenpairs $\left(\mu_{i}, \boldsymbol{u}_{i}\right)$ of $K$ with the eigenpairs $\left(\tilde{\mu}_{i}, \tilde{\boldsymbol{u}}_{i}\right)$ of $\tilde{K}$ by

$$
\mu_{i} \approx \frac{n}{s} \tilde{\mu}_{i}, \quad \boldsymbol{u}_{i} \approx \sqrt{\frac{s}{n}} \frac{1}{\tilde{\mu}_{i}} C \tilde{\boldsymbol{u}}_{i},
$$

for $i=1, \ldots, s$.

Relationship (7) leads to the following rank-s approximation of $K$ :

$$
K \approx \sum_{i=1}^{s} \mu_{i} \boldsymbol{u} \boldsymbol{u}^{\mathrm{T}}=C \tilde{K}^{-1} C^{\mathrm{T}} .
$$

Here, computation of $K^{-1} \widehat{U} \widehat{C}$ is required to obtain $\widehat{B}$. We consider using the pseudo-inverse of $C \tilde{K}^{-1} C^{\mathrm{T}}$ instead of $K^{-1}$. By using the thin QR decomposition of $C$,

$$
C=Q R, \quad Q \in \mathbb{R}^{n \times s}, R \in \mathbb{R}^{s \times s},
$$

and equation (8) is represented as

$$
K \approx Q R \hat{K}^{-1} R^{\mathrm{T}} Q^{\mathrm{T}} .
$$

By introducing the singular value decomposition of $R \hat{K}^{-1} R^{\mathrm{T}}$

$$
R \hat{K}^{-1} R^{\mathrm{T}}=\left[\begin{array}{ll}
U & U^{\prime}
\end{array}\right]\left[\begin{array}{cc}
\Sigma & \\
& O
\end{array}\right]\left[\begin{array}{c}
U^{\mathrm{T}} \\
U^{\prime \mathrm{T}}
\end{array}\right],
$$




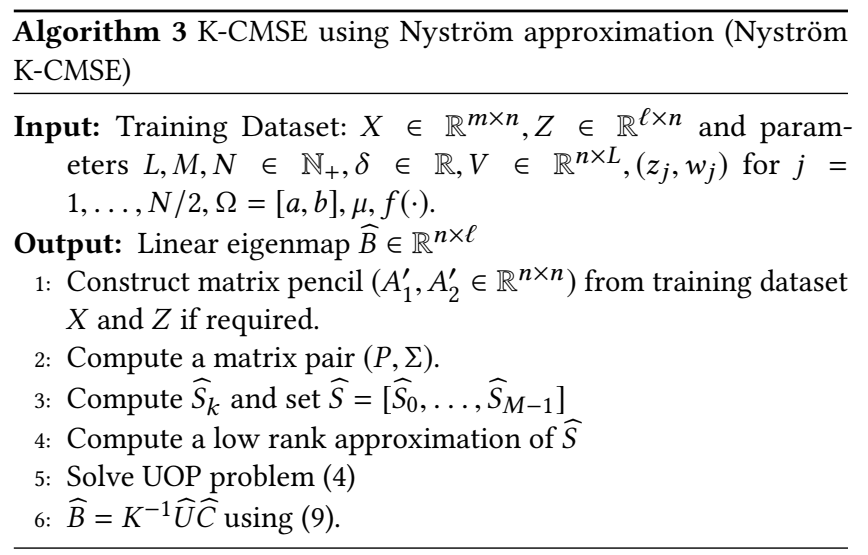

we approximate $K$ as:

$$
K \approx Q\left[\begin{array}{ll}
U & U^{\prime}
\end{array}\right]\left[\begin{array}{ll}
\Sigma & \\
& O
\end{array}\right]\left[\begin{array}{c}
U^{\mathrm{T}} \\
U^{\prime \mathrm{T}}
\end{array}\right] Q^{\mathrm{T}} .
$$

Thus, the inverse is approximated by

$$
K^{-1} \approx P \Sigma^{-1} P^{\mathrm{T}}
$$

where $P=Q U$.

Algorithm 3 summarizes K-CMSE using the Nyström approximation, Nyström K-CMSE.

\subsection{Splitting Interval into Sub-intervals}

Consider the case that $L$ and/or $M$ cannot be set large enough for computing $\widehat{S}$ due to, e.g., the memory constraints of compute nodes.

Consider splitting the interval $\Omega$ into disjoint sub-intervals as

$$
\Omega=\Omega_{1} \cup \cdots \cup \Omega_{N_{\mathrm{I}}} .
$$

Let $S^{(i)}, i=1, \ldots, N_{\mathrm{I}}$ be $S$ generated by equation (2) in a subinterval $\Omega_{i}$. The target subspace of whole $\Omega$ is then represented with $S^{(i)}$ by

$$
\mathcal{S}_{\Omega}=\mathcal{R}\left(\left[S^{(1)} \ldots S^{\left(N_{\mathrm{I}}\right)}\right]\right) .
$$

Thus, we can compute the target subspace by splitting $\Omega$ into subintervals. In this case, it may be necessary to compute the low-rank approximation of $S$.

\subsection{Efficient Parallel Implementation}

The most time-consuming part of Nyström K-CMSE is to solve linear systems:

$$
\left(z_{j} A_{2}^{\prime}-A_{1}^{\prime}\right) Y_{j}=A_{2}^{\prime} V, \quad j=1, \ldots, \frac{N}{2} .
$$

By using the natural parallelism of computing $S$, the computation time can be reduced drastically.

Computing $\widehat{S}$ in (K-)CMSE has the following hierarchical parallelism:

- If $\Omega$ is split into sub-intervals, the computation of each subinterval can be performed independently. This is called parallelism of intervals and is referred to as the "Top Layer".

- In each interval, the linear systems (10) can be solved simultaneously because its coefficient matrix is independent. This

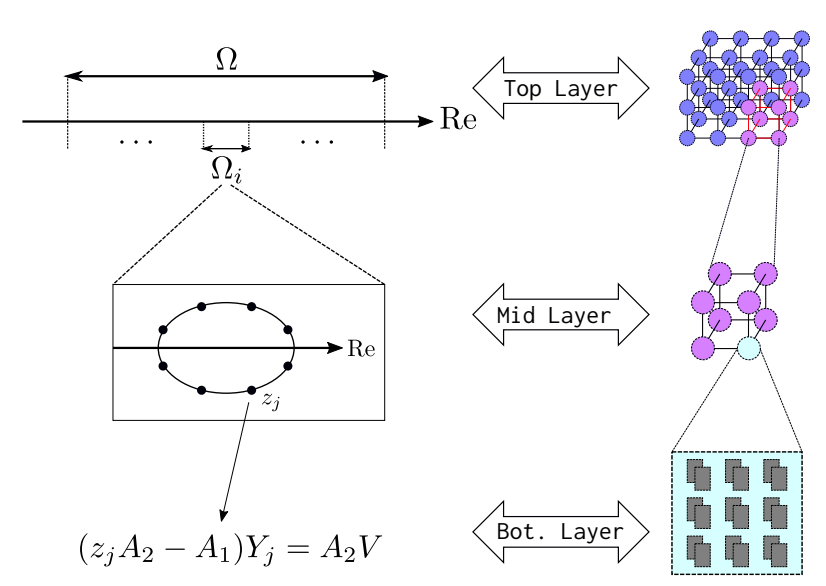

Figure 1: Hierarchical parallelism of (K-)CMSE method. Blue, purple, and cyan parts represent MPI_COMM_WORLD, toplevel communicator, and middle-level communicator, respectively.

is called parallelism of integral points and is referred to as the "Middle Layer".

- In each linear system, we can use a parallel linear solver. This is called parallelism of linear solvers and is referred to as the "Bottom Layer".

To exploit the parallelism described above, we use MPI and assign MPI ranks as follows:

(1) We split MPI_COMM_WORLD into sub-communicators associated with Top Layer parallelism and assign each sub-communicator to sub-interval(s). A sub-communicator of this level is called a top-level communicator.

(2) Using a distributed memory parallel implementation of a linear solver, e.g., MUMPS, we further split top-level communicator(s) into sub-communicators. A sub-communicator of this level is called a middle-level communicator.

(3) For each linear system, we assign a middle-level communicator or a rank of the top-level communicator.

For each sub-interval, we can solve $N / 2$ linear systems at most in N/2-parallel, and each linear system can be solved using a distributed parallel linear solver. By assuming each parallel linear solver is executed with $P_{\mathrm{LS}}$ MPI processes, the maximum number of processes we can use is $N_{\mathrm{I}} \cdot N / 2 \cdot P_{\mathrm{LS}}$. Figure 1 summarizes the relationships in the hierarchical algorithm and the MPI rank assignment described above. The proposed parallel implementation is shown in Algorithm 4.

\section{NUMERICAL EXPERIMENT}

To evaluate the proposed parallel implementation, we performed some numerical experiments. Experiment I verifies the reduction in computation time by the Nyström approximation. Experiment II evaluates strong scalability of the proposed implementation. Note that only the Middle Layer of the hierarchical parallelism is evaluated in Experiment II. The bottom Layer is parallelized by using 


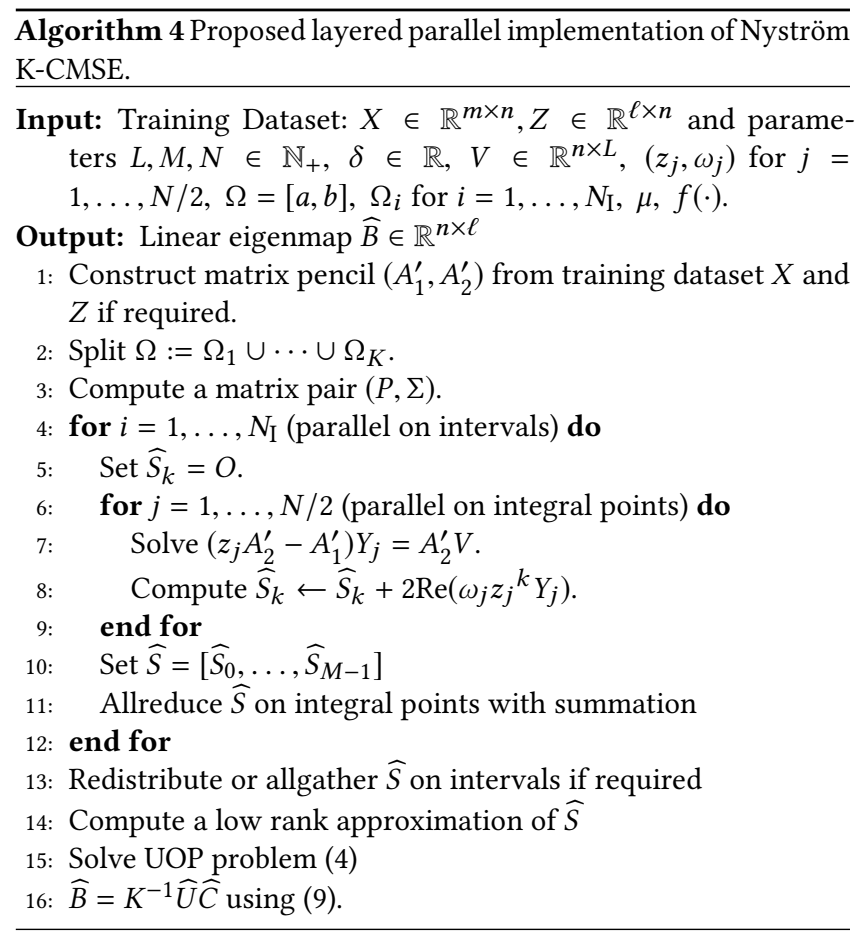

Table 1: Hardware specification of a compute node of Oakforest-PACS.

\begin{tabular}{ll}
\hline Processor & Intel Xeon Phi 7250 ×1 \\
Memory & DDR4-2400 96 GB + MCDRAM 16 GB \\
Interconnect & Intel Omni-Path $12.5 \mathrm{~GB} / \mathrm{s}$ \\
\hline
\end{tabular}

a multi-threaded linear solver. The number of threads is kept constant for all the experiments. Evaluation of the distributed parallel performance of Top and Bottom Layers is a work for the future.

\subsection{Experiment Setting}

The experiments were performed on Oakforest-PACS, a Knights Landing Xeon Phi cluster operated by Joint Center for Advanced High Performance Computing (JCAHPC), using up to 16 nodes. Table 1 shows the hardware specification of a compute node. In the experiments, MCDRAM is used with the flat mode, and the number of OpenMP threads is 134 with KMP_AFFINITY=balanced.

We implemented K-CMSE and proposed implementation in $\mathrm{C}++$ using MPI. Table 2 shows the software environment used for the experiments. The computation time is measured by steady_clock in std: : chrono, a software timer provided by the $\mathrm{C}++11$ standard library. In the experiments, the following parts were excluded from the elapsed time: loading the dataset, generating matrices $A_{1}^{\prime}$ and $A_{2}^{\prime}$, and post-processing of dimensionality reduction, e.g., classification using low-dimensional representation. The computation of $\widehat{S}$ is performed by z-Pares [4], a distributed parallel software of complex moment-based eigensolvers. Because z-Pares returns the eigenpairs extracted from $\widehat{S}$, we modified it to return $\widehat{S}$ instead of the eigenpair. We use Intel MKL Inspector-executor Sparse BLAS Routines for
Table 2: Software environment for experiments.

\begin{tabular}{ll}
\hline Compiler & Intel Compiler 19.0.4.243 \\
& GCC 8.3.0 (Header only) \\
Compilation option & -03 -qopenmp -axMIC-AVX512 \\
MPI & Intel MPI 2019 Update 4 \\
Dependency & Intel MKL 2019.0 Update 4 \\
& z-Pares C++ interface \\
\hline
\end{tabular}

sparse matrix-dense matrix multiplication and Intel MKL PARDISO with $\mathrm{mtype}=6$ as the linear solver for solving the linear systems (10).

We used the MNIST hand written digit classification problem [8] as the benchmark problem. We set the original dataset $X$ as randomly chosen 40,000 images of 60,000 training images. $\ell$ is set as 10 , the same number as the number of classes, and the ground truth $Z$ is given by

$$
z_{i j}= \begin{cases}1 & \left(\text { if } x_{j} \text { is classified as } i\right) \\ -1 & \text { (otherwise })\end{cases}
$$

We use the Gaussian kernel as the kernel function. Matrices $A_{1}^{\prime}$ and $A_{2}^{\prime}$ are the same matrices as K-LPP [5] with $k$-nearest neighbor $(k=7)$. The landmark of the Nyström approximation consists of 40 randomly selected samples in the original dataset $X$. The parameters of K-CMSE are set as $N=32, M=8, L=75, \delta=10^{-15}$, and $\mu=0.5$. The input matrix $V$ is generated from the uniform distribution $[-1,1)$ computed by Mersenne Twister. We set $\Omega=[0,1]$, and the integral points are set on an ellipse with center $\gamma=1 / 2$, major axis $\rho=1 / 2$, and aspect ratio $\alpha=0.1$ :

$$
z_{j}=\gamma+\rho\left(\cos \left(\theta_{j}\right)+\mathrm{i} \alpha \sin \left(\theta_{j}\right)\right), \quad \theta_{j}=\frac{2 \pi}{N}\left(j-\frac{1}{2}\right),
$$

for $j=1, \ldots, N / 2$. The corresponding integral weights are

$$
\omega_{j}=\frac{\rho}{N}\left(\alpha \cos \left(\theta_{j}\right)+\mathrm{i} \sin \left(\theta_{j}\right)\right)
$$

for $j=1, \ldots, N / 2$. The weight function $f(\cdot)$ is defined as $f(\lambda)=$ $1 /(1-\lambda)^{2}$. For solving the UOP problem (4), we use an iterative method proposed by Zhao et al. [16] and the iteration count is set as 10 .

\subsection{Experiment I: Use of Nyström Approximation}

In Experiment I, we verify that the computation time of K-CMSE is reduced by the Nyström approximation. The naive implementation of K-CMSE and the proposed implementation, Nyström K-CMSE, were executed on one node. The result of Experiment I is shown in Table 3.

In Table 3, Gen_K, SVD_K, Compute_S, LRA, Form_UOP, and Solve_UOP represent the computation times of generating the Gram matrix $K$, singular value decomposition of $K$, forming $\widehat{S}$, $A_{2}$-orthogonal low rank approximation of $\widehat{S}$, forming the matrices of the UOP problem, and solving the UOP problem, respectively. Note that Gen_K in Nyström K-CMSE denotes the generation of a matrix pair that approximates the kernel matrix.

Table 3 shows the times of K-CMSE for computing the singular value decomposition of $K$. It is observed that Nyström K-CMSE is 
Table 3: Computation times of K-CMSE and Nyström KCMSE in seconds.

\begin{tabular}{rrr}
\hline & K-CMSE & Nyström K-CMSE \\
\hline Gen_K & 28.446 & 0.229 \\
SVD_K & 7649.441 & --- \\
Compute_S & 103.093 & 92.254 \\
LRA & 4.595 & 2.184 \\
Form_UOP & 0.797 & 0.325 \\
Solve_UOP & 5.638 & 3.314 \\
\hline
\end{tabular}

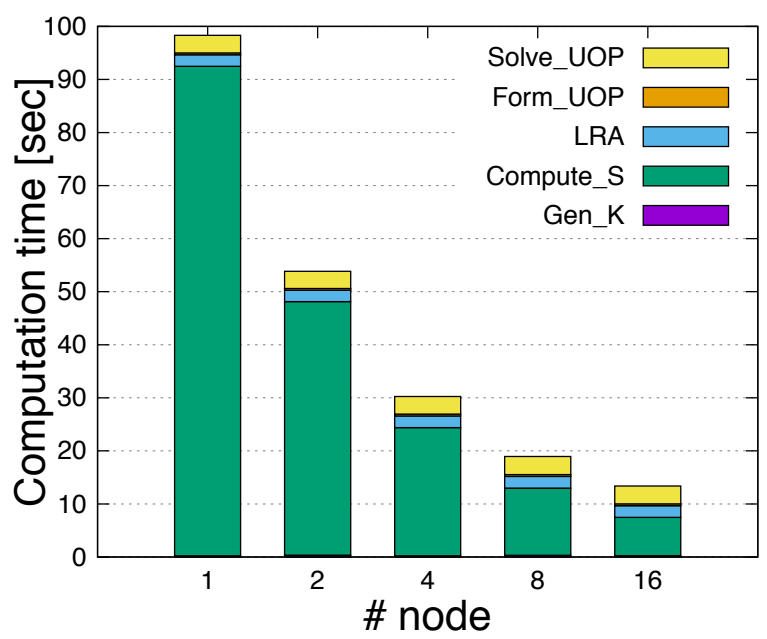

Figure 2: Computation times of Nyström K-CMSE on up to 16 nodes.

much faster than K-CMSE because of the absence of the singular value decomposition of $K$ itself. This result indicates that when $n$ is large, handling $K$ directly is impractical, and an approximation method such as the Nyström approximation is required.

\subsection{Experiment II: Strong Scalability}

In Experiment II, we evaluate the parallel performance of the proposed implementation by varying the number of nodes: $1,2,4,8$, and 16. The number of MPI ranks per node is fixed to one. The results of Experiment II are shown in Figures 2, 3, and 4.

In Figure 2, each entry has the same meaning as that in Table 3. The entries in Figure 3 are the same as those in Figure 2. In Figure 4, Total denotes the sum of Gen_K, Compute_S, LRA, Form_UOP, and Solve_UOP.

Figures 2 and 4 show the time for computing $\widehat{S}$ scales almost linearly. This shows that the computation time of the most dominant part of Nyström K-CMSE can be reduced by exploiting Middle Layer of the hierarchical parallelism. It is also for the computation time of Nyström K-CMSE, high scalability is achieved. However, Figure 3 shows that the ratios of the computation times of the low rank approximation of $\widehat{S}$ and the UOP problem solution increase when the number of nodes for parallel execution is increased. Thus, distributed parallel implementation of these computation is required to further improve the parallel efficiency.

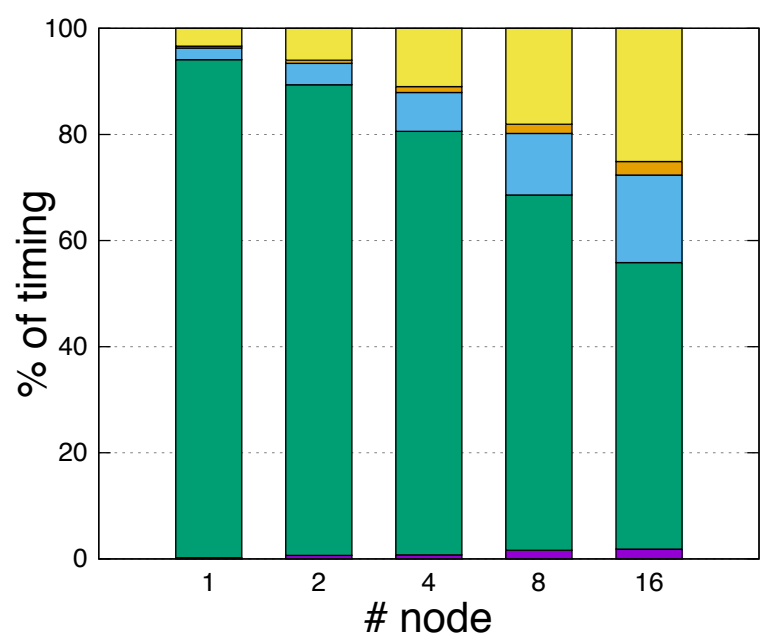

Figure 3: Ratio of computation time of Nyström K-CMSE on up to 16 nodes.

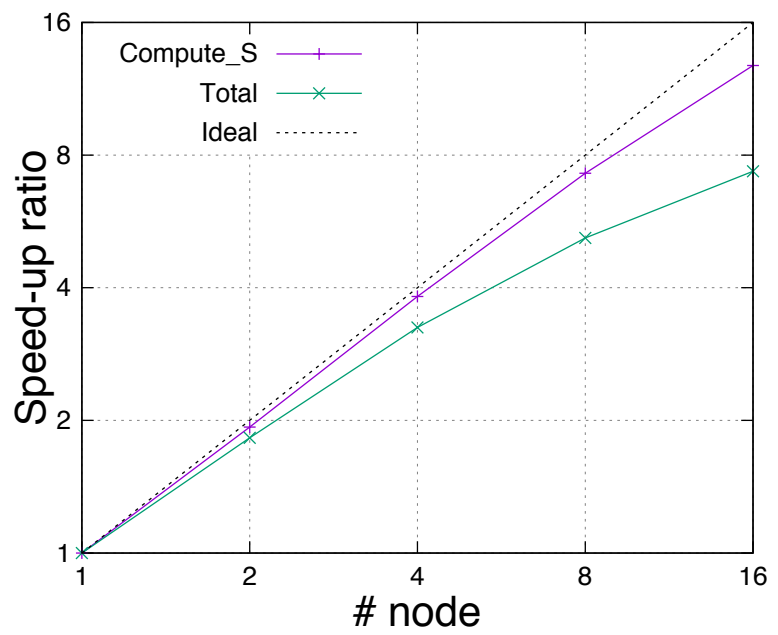

Figure 4: Speed-up ratio of Compute_S and total Nyström K-CMSE with respect to 1 node.

\section{CONCLUSION}

In this paper, we proposed a practical parallel implementation of K-CMSE. The proposed implementation uses the Nyström approximation to reduce the computational and space complexity needed to handle the Gram matrix. The proposed implementation is also based on the hierarchical parallelism in constructing the complex moment-based subspace to achieve high parallel scalability. In the numerical experiments, the Nyström approximation reduces the computational complexity more successfully than the naive implementation. In addition, the proposed implementation scales well when the Middle Layer of the hierarchical parallelism achieved by constructing the complex moment-based subspace.

Because we evaluated only distributed parallelism of the Middle Layer, in future, we will perform another experiment using the Top and Bottom layers of the hierarchical parallelism. We will also 
evaluate the classification accuracy of Nyström K-CMSE, because introducing the Nyström approximation drops information from the Gram matrix $K$.

\section{ACKNOWLEDGMENTS}

This research (in part) used the computational resources of OakforestPACS provided by Multidisciplinary Cooperative Research Program in Center for Computational Sciences, University of Tsukuba. This research is supported by the New Energy and Industrial Technology Development Organization (NEDO) and the Japan Society for the Promotion of Science (JSPS), Grants-in-Aid for Scientific Research (Nos. 17K12690, 18H03250, 19K20280, and 19KK0255).

\section{REFERENCES}

[1] Lars Eldén and Haesun Park. 1999. A Procrustes problem on the Stiefel manifold. Numerische Mathematik 82, 4 (1999), 599-619. https://doi.org/10.1007/ s002110050432

[2] Ronald A. Fisher. 1936. The use of multiple measurements in taxonomic problems Annals of Eugenics 7, 2 (1936), 179-188. https://doi.org/10.1111/j.1469-1809.1936. tb02137.x

[3] Keinosuke Fukunaga. 1990. Introduction to Statistical Pattern Recognition (second ed.). Academic Press Professional, Inc., San Diego, CA, USA.

[4] Yasunori Futamura. 2014. z-Pares: Parallel Eigenvalue Solver. https://zpares.cs. tsukuba.ac.jp/

[5] Xiaofei He and Partha Niyogi. 2004. Locality preserving projections. In Advances in Neural Information Processing Systems, Vol. 16. MIT Press, Cambridge, MA, USA, 153-160.

[6] Akira Imakura, Momo Matsuda, Xiucai Ye, and Tetsuya Sakurai. 2019. Complex moment-based supervised eigenmap for dimensionality reduction. In Proceedings of the AAAI Conference on Artificial Intelligence, Vol. 33. AAAI Press, Palo Alto,
CA, USA, 3910-3918. https://doi.org/10.1609/aaai.v33i01.33013910

[7] Shigeru Iwase, Yasunori Futamura, Akira Imakura, Tetsuya Sakurai, and Tomoya Ono. 2017. Efficient and scalable calculation of complex band structure using Sakurai-Sugiura method. In Proceedings of the International Conference for High Performance Computing, Networking, Storage and Analysis (Denver, Colorado) (SC '17). Association for Computing Machinery, New York, NY, USA, Article 40, 12 pages. https://doi.org/10.1145/3126908.3126942

[8] Yann LeCun, Léon Bottou, Yoshua Bengio, and Patrick Haffner. 1998. Gradientbased learning applied to document recognition. Proceedings of the IEEE 86, 11 (1998), 2278-2324. https://doi.org/10.1109/5.726791

[9] Xuelong Li, Mulin Chen, Feiping Nie, and Qi Wang. 2017. Locality adaptive discriminant analysis. In Proceedings of the 26th International foint Conference on Artificial Intelligence (Melbourne, Australia) (IFCAI'17). AAAI Press, Palo Alto, CA, USA, 2201-2207. https://doi.org/10.24963/ijcai.2017/306

[10] Haesun Park. 1991. A parallel algorithm for the unbalanced orthogonal procrustes problem. Parallel Computing 17, 8 (1991), 913-923. https://doi.org/10.1016/S01678191(05)80075-4

[11] Karl Pearson. 1901. LIII. On lines and planes of closest fit to systems of points in space. The London, Edinburgh, and Dublin Philosophical Magazine and fournal of Science 2, 11 (1901), 559-572. https://doi.org/10.1080/14786440109462720

[12] Tetsuya Sakurai and Hiroshi Sugiura. 2003. A projection method for generalized eigenvalue problems using numerical integration. Fournal of Computational and Applied Mathematics 159, 1 (2003), 119-128. https://doi.org/10.1016/S03770427(03)00565-X

[13] Bernhard Schölkopf, Alexander Smola, and Klaus-Robert Müller. 1998. Nonlinear component analysis as a kernel eigenvalue problem. Neural Computation 10, 5 (1998), 1299-1319. https://doi.org/10.1162/089976698300017467

[14] Masashi Sugiyama. 2007. Dimensionality reduction of multimodal labeled data by local Fisher discriminant analysis. Fournal of Machine Learning Research 8, 37 (2007), 1027-1061.

[15] Christopher K. I. Williams and Matthias Seeger. 2001. Using the Nyström Method to Speed Up Kernel Machines. In Advances in Neural Information Processing Systems, Vol. 13. MIT Press, Cambridge, MA, USA, 682-688.

[16] Haifeng Zhao, Zheng Wang, and Feiping Nie. 2016. Orthogonal least squares regression for feature extraction. Neurocomputing 216 (2016), 200-207. https: //doi.org/10.1016/j.neucom.2016.07.037 